\title{
Chemotherapy reduces PARP1 in cancers of the ovary: implications for future clinical trials involving PARP inhibitors
}

\author{
Maud Marques ${ }^{1 \dagger}$, Marie-Claude Beauchamp ${ }^{1 \dagger}$, Hubert Fleury ${ }^{2}$, Ido Laskov ${ }^{1,3}$, Sun Qiang ${ }^{1}$, Manuela Pelmus ${ }^{4}$,
} Diane Provencher ${ }^{2,5,6}$, Anne-Marie Mes-Masson ${ }^{5,6}$, Walter H. Gotlieb ${ }^{1,3^{*}}$ and Michael Witcher ${ }^{1 *}$

\begin{abstract}
Background: PARP inhibitors have shown promising clinical results in cancer patients carrying BRCA1/2 mutations. Their clinical efficacy could logically be influenced by PARP1 protein levels in patient tumors.

Methods: We screened three cohorts of patients with ovarian cancer, totaling 313 samples, and evaluated PARP1 protein expression by immunohistochemistry with further validation by western blotting.

Results: We observed that up to $60 \%$ of tumors showed little PARP1 protein expression. In serous ovarian tumors, comparing intratumoral PARP1 expression between chemo-naïve and post-chemotherapy patients revealed a decrease in intratumoral PARP1 following chemotherapy in all three cohorts (immunohistochemistry: $p<0.001, \mathrm{n}=239$; western blot: $p=0.012, n=74$ ). The findings were further confirmed in a selection of matched samples from the same patients before and after chemotherapy.

Conclusion: Our data suggest that patients should be screened for PARP1 expression prior to therapy with PARP inhibitors. Further, the observed reduction of intratumoral PARP1 post-chemotherapy suggests that treating chemo-naïve patients with PARP inhibitors prior to the administration of chemotherapy, or concurrently, might increase the responsiveness to PARP1 inhibition. Thus, a change in the timing of PARP inhibitor administration may be warranted for future clinical trials.
\end{abstract}

Keywords: Chemotherapy, PARP inhibitors, PARP1, ovarian cancer

\section{Background}

Unlike breast cancer, the overall survival of patients with ovarian cancer has shown only slight improvement over the past 30 years [1], with high-grade serous cancers (HGSC) of the ovary being the most deadly gynecologic cancer. While many patients with HGSC respond to first-line chemotherapy, recurrence and drug resistance occur in the vast majority of cases [2]. Identification of key biomarkers or predictors for response to one or a combination of specific antitumor drugs seems essential.

Over the past 5 years, clinical trials using various PARP inhibitors on patients with $B R C A 1 / 2$ mutations $[3,4]$ have

\footnotetext{
* Correspondence: walter.gotlieb@mcgill.ca; michael.witcher@mcgill.ca ${ }^{\dagger}$ Equal contributors

'Lady Davis Institute for Medical Research, Jewish General Hospital, 3755

Côte Ste, Catherine Road, Montréal, QC H3T 1E2, Canada

Full list of author information is available at the end of the article
}

end of the article shown promising results [5]. Inherent defects in the homologous recombination DNA repair pathway in $B R C A$-deficient tumors coupled with the inhibition of PARP1 lead to a synthetic lethality culminating in cancer cell death [6]. It is estimated that almost half of patients with HGSC bear defects in homologous recombination [7], spurring the onset of clinical trials to test PARP inhibitors in this population.

The reported response rate for BRCA-deficient HGSC in the ovary treated with PARP inhibitors ranges between 25 and $53 \%$ [5, 8-12]. In these published studies, enrolled patients had received several cycles of adjuvant chemotherapy prior to PARP inhibitors.

In this study, we evaluated the effect of chemotherapy on PARP1 expression in solid tumors.

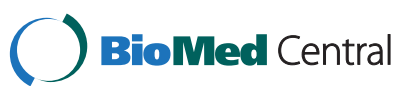

(c) 2015 Marques et al. Open Access This article is distributed under the terms of the Creative Commons Attribution 4.0 International License (http://creativecommons.org/licenses/by/4.0/), which permits unrestricted use, distribution, and reproduction in any medium, provided you give appropriate credit to the original author(s) and the source, provide a link to the Creative Commons license, and indicate if changes were made. The Creative Commons Public Domain Dedication waiver (http://creativecommons.org/publicdomain/zero/1.0/) applies to the data made available in this article, unless otherwise stated. 


\section{Methods}

\section{Patients}

Neo-adjuvant chemotherapy consisted of three to four combined courses of a platinum-based agent (carboplatin) and paclitaxel given prior to surgery. Changes to this protocol were made depending on the patient's condition, response, compliance, and side effects. The patients were considered responsive to this regimen if recurrence occurred more than 6 months after the last chemotherapy treatment.

\section{Training cohort: immunohistochemistry (IHC) analysis}

Tumor samples were obtained from 69 patients who had undergone surgery and chemotherapy in the Division of Gynecologic Oncology of McGill University's Jewish General Hospital from 2006 to 2014 (Table 1). Diagnosis was based on final pathology, determined by an independent gynecologic pathologist. The tumors were staged according to the International Federation of Gynecology and Obstetrics (FIGO) stage and histologically classified and graded according to the World Health Organization grade. Tissue selection criteria for this study were based on chemotherapy-naïve and chemotherapy-treated patients.

Table 1 Training cohort: clinical characteristics of patients for immunohistochemistry experiment

\begin{tabular}{|c|c|c|}
\hline \multicolumn{2}{|l|}{ Patients } & Number (\%) \\
\hline \multicolumn{2}{|l|}{ Number } & 69 \\
\hline \multicolumn{2}{|c|}{ Age (years), mean (range) } & $61.9(36-86)$ \\
\hline Histology & Serous & $69(100)$ \\
\hline \multirow[t]{4}{*}{ Stage } & I & $6(9)$ \\
\hline & $\|$ & $6(9)$ \\
\hline & III & $51(74)$ \\
\hline & IV & $5(7)$ \\
\hline \multirow[t]{3}{*}{ Grade } & 1 & $2(3)$ \\
\hline & 2 & $3(4)$ \\
\hline & 3 & $57(83)$ \\
\hline \multirow[t]{2}{*}{ Chemotherapy } & Neo-adjuvant & $35(51)$ \\
\hline & Adjuvant & $32(46)$ \\
\hline \multirow[t]{2}{*}{ Surgery } & Not robotic & $32(46)$ \\
\hline & Robotic & $35(51)$ \\
\hline \multicolumn{2}{|l|}{ Residual disease } & $4(6)$ \\
\hline \multirow[t]{2}{*}{ Response } & Responsive & $41(59)$ \\
\hline & Resistant & $19(28)$ \\
\hline \multirow[t]{2}{*}{ Recurrence } & Number & $41(59)$ \\
\hline & Mean time (range) & 17.9 months (3-84) \\
\hline \multirow[t]{2}{*}{ Deceased } & Number & $21(30.4)$ \\
\hline & Mean time (range) & 27.9 months(10-72) \\
\hline Follow-up & Mean time (range) & 36.6 months (7-111) \\
\hline
\end{tabular}

Validation cohort 1: Tissue microarray (TMA)

Tumor samples were obtained from 170 patients who had undergone surgery for ovarian cancer at the Department of Gynecologic Oncology Centre Hospitalier de l'Université de Montréal (Table 2). An independent pathologist reviewed tumor histopathology, grade, and chemotherapy status of each patient. Tissue selection criteria for this study were based on chemotherapy-naïve and chemotherapy-treated patients. Samples were collected between 1993 and 2014.

\section{Validation cohort 2: western blot analysis}

Tissue samples were included from 74 patients (mean age 60.7, range 24-87) with ovarian cancer who had undergone surgery and chemotherapy in the Jewish General Hospital from 2004 to 2013 (Table 3). The patient's course of treatment was determined by a team of gynecologic oncologists in accordance with the National Comprehensive Cancer Network guidelines.

\section{Ethical approval}

The research complied with all requirements of the Helsinki Declaration. Tissue samples were taken from the

Table 2 Validation cohort 1: clinical characteristics of patients for tissue microarray experiment

\begin{tabular}{|c|c|c|}
\hline \multicolumn{2}{|l|}{ Patients } & Number (\%) \\
\hline \multicolumn{2}{|l|}{ Number } & 170 \\
\hline \multicolumn{2}{|c|}{ Age (years), mean (range) } & 52 (39-93) \\
\hline \multirow[t]{2}{*}{ Histology } & Serous & $150(88.2)$ \\
\hline & Non serous & $20(11.8)$ \\
\hline \multirow[t]{4}{*}{ Stage } & I & $7(4.1)$ \\
\hline & $\|$ & $11(6.4)$ \\
\hline & III & $129(72.9)$ \\
\hline & IV & $21(12.3)$ \\
\hline \multirow[t]{3}{*}{ Grade } & 1 & $6(3.5)$ \\
\hline & 2 & $22(12.9)$ \\
\hline & 3 & $136(80)$ \\
\hline \multirow[t]{2}{*}{ Chemotherapy } & Neo-adjuvant & $85(50)$ \\
\hline & Adjuvant & $85(50)$ \\
\hline \multirow[t]{3}{*}{ Residual disease } & $\leq 1 \mathrm{~cm}$ & $87(51.2)$ \\
\hline & $\geq 1 \mathrm{~cm}$ & $57(33.5)$ \\
\hline & Not determined & $26(15.3)$ \\
\hline \multirow[t]{2}{*}{ Response } & Responsive & $123(72.3)$ \\
\hline & Resistant & $47(27.7)$ \\
\hline \multirow[t]{2}{*}{ Recurrence } & Number & $128(75.3)$ \\
\hline & Mean time (range) & 20 months (2-76) \\
\hline \multirow[t]{2}{*}{ Deceased } & Number & $105(61.8)$ \\
\hline & Mean time (range) & 47 months (2-156) \\
\hline Follow up & Mean time (range) & 43 months (2-73) \\
\hline
\end{tabular}


Table 3 Validation cohort 2: clinical characteristics of patients for western blot experiment

\begin{tabular}{|c|c|c|}
\hline \multicolumn{2}{|l|}{ Patients } & Number (\%) \\
\hline \multicolumn{2}{|l|}{ Number } & 74 \\
\hline \multicolumn{2}{|c|}{ Age(y), mean(range) } & $60.7(24-87)$ \\
\hline \multirow[t]{2}{*}{ Histology } & Serous & $48(64.9)$ \\
\hline & Non Serous & $26(35.1)$ \\
\hline \multirow[t]{4}{*}{ Stage } & I & $11(16.4)$ \\
\hline & $\|$ & $5(7.5)$ \\
\hline & III & $42(62.7)$ \\
\hline & IV & $9(13.4)$ \\
\hline \multirow[t]{3}{*}{ Grade } & 1 & $9(16.9)$ \\
\hline & 2 & $5(9.4)$ \\
\hline & 3 & 39 (73.6) \\
\hline \multirow[t]{2}{*}{ Chemotherapy } & Neo-adjuvant & $34(46)$ \\
\hline & Adjuvant & $25(34)$ \\
\hline \multirow[t]{3}{*}{ Surgery } & Laparotomy & $30(46)$ \\
\hline & Laparoscopy & $2(3)$ \\
\hline & Robotic & $33(51)$ \\
\hline \multicolumn{2}{|l|}{ Residual disease } & $8(10.8)$ \\
\hline \multirow[t]{2}{*}{ Response } & Responsive & $33(75)$ \\
\hline & Resistant & $11(25)$ \\
\hline \multirow[t]{2}{*}{ Recurrence } & Number & $27(36.5)$ \\
\hline & Mean time (range) & 9.6 months (3-29) \\
\hline \multirow[t]{2}{*}{ Deceased } & Number & $21(31.3)$ \\
\hline & Mean time (range) & 26.5 months (4-88) \\
\hline Follow-up & Mean time (range) & 31.4 months (7-107) \\
\hline
\end{tabular}

gynecologic oncology tumor bank and approved by the Jewish General Hospital (protocol\#14-025). Ethic approval was obtained from the Jewish General Hospital Research Ethics Committee (protocol\#03-041). All patients participating in this study gave informed written consent.

\section{PARP1 antibody validation}

PARP1 antibody (Clone B-10, sc74470, Santa Cruz Biotechnology, Inc. Dallas, TX, USA) specificity was validated for both western blot and IHC (Additional file 1: Figure S1). Epithelial ovarian cancer cells OVCAR8 were infected using shctl or shPARP1. After 5 days, cells were collected and either fixed in formalin for IHC analysis or proteins were extracted for western blot analysis.

\section{Immunohistochemistry}

Biopsies and surgical specimens were formalin-fixed at the Pathology Facility (Jewish General Hospital) for $12 \mathrm{~h}$ at room temperature, processed, and paraffin-embedded. IHC staining was performed at the Segal Cancer Center Research Pathology Facility as previously described [13]. Briefly, tissue samples were cut at $4 \mu \mathrm{m}$, placed on
SuperFrost/Plus slides (Fisher Scientific, Pittsburgh, PA, USA), and dried overnight at $37^{\circ} \mathrm{C}$. The slides were then loaded onto the Discovery XT Autostainer (Ventana Medical Systems, Inc. Tucson, AZ, USA). All solutions used for automated IHC were from Ventana Medical Systems unless otherwise specified. Slides underwent deparaffinization with the EZ PREP solution (Ref\# 950-100), followed by heat-induced epitope retrieval with Cell Conditioning Solution 1, pH 8.0 (Ref\# 950-224), in standard conditions $\left(60 \mathrm{~min}\right.$ at $95^{\circ} \mathrm{C}$ ). Immunostaining for PARP1 was performed online using a heat protocol. Briefly, mouse monoclonal anti-PARP1 (B-10, sc74470, Santa Cruz Biotechnology, Inc.) diluted at 1:100 in antibody diluent (Ref\# 251-018) was manually applied for $32 \mathrm{~min}$ at $37{ }^{\circ} \mathrm{C}$, then PARP1 protein was detected using Omnimap anti-Mouse HRP Kit (Ref\# 760-4310) and ChromoMapDAB (Ref\# 760-159). Slides were counterstained with hematoxylin for $4 \mathrm{~min}$; blued with Bluing Reagent for 4 min; removed from the autostainer; washed in warm soapy water (Dawn) dehydrated through graded alcohols; cleared in xylene; and mounted with Permount. The percentage of tumor cells stained was assessed by an experienced pathologist (MP, assisted by MM, M-CB and IL) and ranked as follow: $0=$ no staining, $1=<25 \%$ staining, $2=25-50 \%, 3=>75 \%$. The intensity of the staining was ranked as follow: $0=$ no staining, $1=$ mild staining, $2=$ moderate staining, 3 = strong staining. Scores for percentage of distribution and intensity were multiplied by each other to reach a final score ranging between 0 and 9 (intensity $\times$ distribution) for comparison analysis. For TMA analysis, the intensity of staining was evaluated by two people blinded to the identification of the cores. More than $80 \%$ of similarity was observed between the two independent scores. Only tumor tissue was taken into account for the score calculation.

\section{Pre-post chemotherapy epithelial ovarian tumor tissue microarray}

A gynecologic pathologist reviewed all samples prechemotherapy and post-chemotherapy to identify the grade and type of ovarian carcinoma. Areas of interest were marked on slides. Two cores of $0.6 \mathrm{~mm}$ for each tissue sample were arrayed onto one recipient paraffin block. This tissue array was composed of cores from 170 epithelial ovarian tumors (two cores per patient sample) and was built on two recipient blocks.

\section{Protein extraction and western blot analysis}

In total, 100 unselected tumor samples from 74 patients (cohort 3) were analyzed, comprising 21 ascites cells pellet, 57 primary tumors, 18 omental tumors, and 4 other tumor tissues. Similar levels of PARP1 protein were observed between the tumor and the omentum isolated from the same patient at the time of surgery (data not 
shown). Snap-frozen tumor tissues were minced and lysed in lysis buffer (25 mM Tris. $\mathrm{HCl} \mathrm{pH} \mathrm{7.6,} 10 \%$ glycerol, $420 \mathrm{mM} \mathrm{NaCl}, 2 \mathrm{mM} \mathrm{MgCl}$, $0.5 \% \mathrm{NP}-40,0.5 \%$ Triton X-100, $1 \mathrm{mM}$ EDTA, protease inhibitor) on ice. Briefly, clarified protein lysates $(50 \mu \mathrm{g})$ were resolved on $6 \%$ and $10 \%$ denaturing SDS-polyacrylamide gels, and transferred to nitrocellulose membranes, similar to as previously described [14]. After blocking in $5 \%$ milk, membranes were probed with primary antibodies specific for BRCA1 (cat\#OP92, Calbiochem, Gibbstown, NJ, USA), PARP1 (sc74470), and $\beta$-actin (Cell Signaling Technology, Whitby, Ontario, Canada). Immunoblotted proteins were visualized using horseradish peroxidaseconjugated secondary antibodies and antigen-antibody complexes were detected using the ECL system (Bio-Rad Laboratories, Inc. Hercules, CA, USA).

\section{Statistical analysis}

Statistical analysis was performed using SPSS (version 22) and R (version 3.1.0). The data were assessed as nonparametric data. A $\chi^{2}$ test was used to analyze the effect of chemotherapy on PARP1 and BRCA1 protein level [15]. We used a two-tailed Mann-Whitney U test to analyze dependency between treatment and PARP1 protein quantity [16]. A two-tailed Wilcoxon signed ranks test was used to evaluate this phenomenon in the same patient before and after chemotherapeutic treatment [15]. Differences with a $p$-value $<0.05$ were considered statistically significant.

\section{Results and discussion}

We first analyzed gene expression $(n=570)$ and reversephase protein analysis $(n=412)$ data made publically available from The Cancer Genome Atlas (TCGA) website for HGSC and found low PARP1 protein levels in a majority of the tumors (Fig. 1), consistent with previous findings in breast tumors [17]. These data prompted us to evaluate PARP1 protein levels in a set of 69 HGSC by IHC (training cohort). Of these, $71 \%$ were characterized as having low PARP1 expression (Fig. 1b,c). This data was

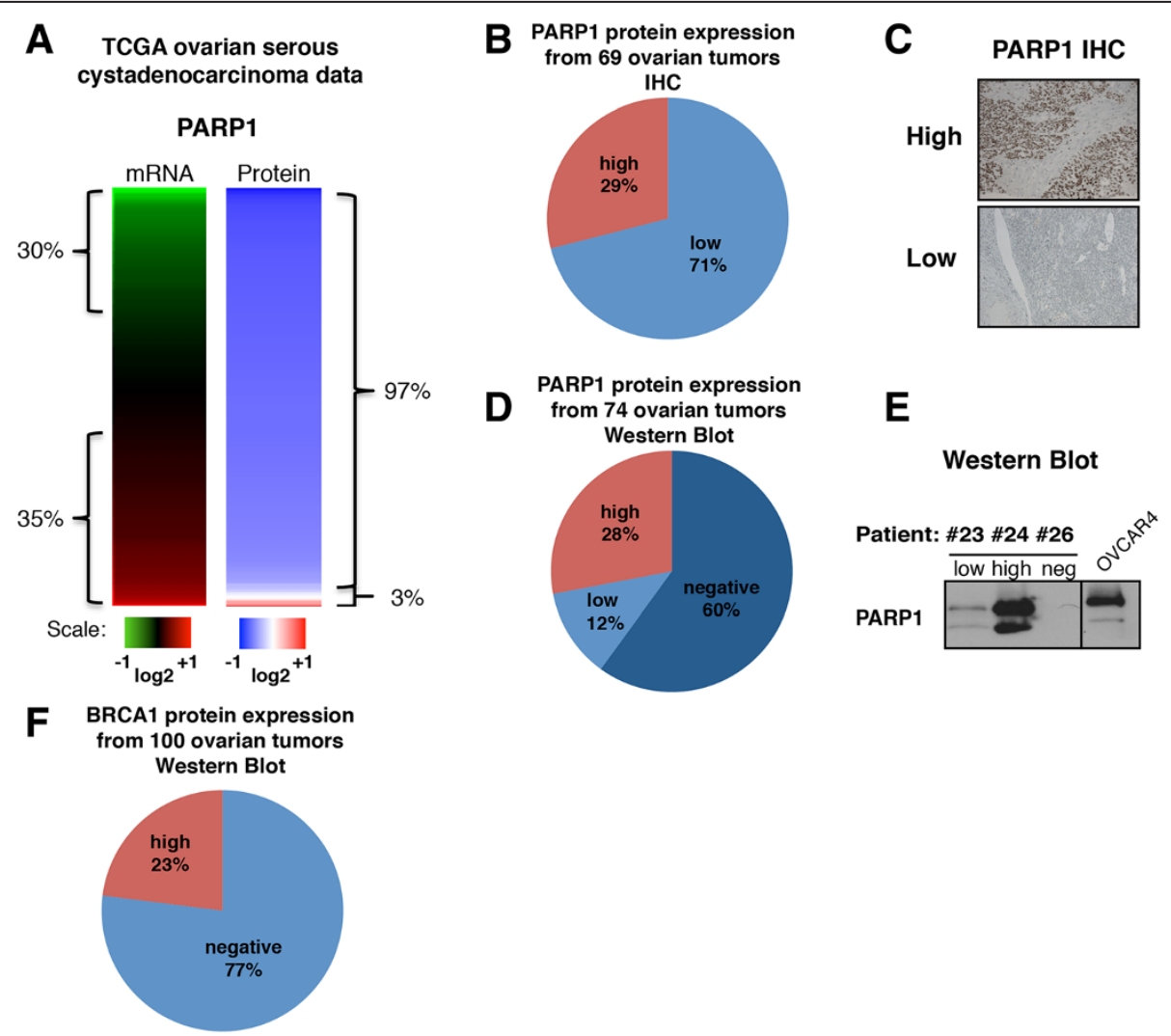

Fig. 1 Low PARP1 protein expression in ovarian tumors. a Analysis of The Cancer Genome Atlas (TCGA) ovarian cystadenocarcinoma database for PARP1 mRNA ( $n=570)$ and protein $(n=412)$ expression levels. Data were used to generate a heat map with red representing high expression level and green low expression level. b Pie chart representing PARP1 protein level as determined by immunohistochemistry (IHC) in 69 HGSC samples (training cohort). c PARP1 representative IHC for each expression category (low and high). $\mathbf{d}$ Pie chart distribution of PARP1 in 100 unselected ovarian tumors from 74 patients (validation cohort 2, several patients had multiple tumors examined) evaluated by western blotting. e PARP1 representative western blot for each expression category (low, high, and negative); OVCAR4 protein extract was used as positive control. f Pie chart representing BRCA1 protein level distribution from 74 patients (100 tumors, validation cohort 2) evaluated by western blotting 


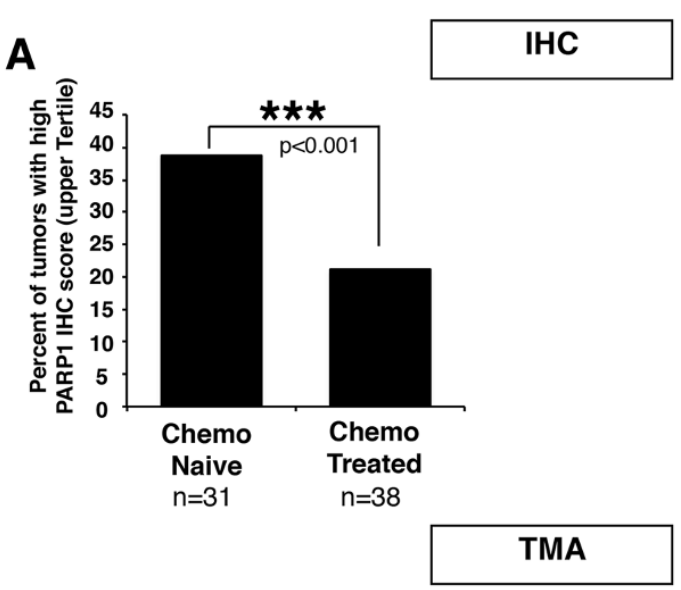

B

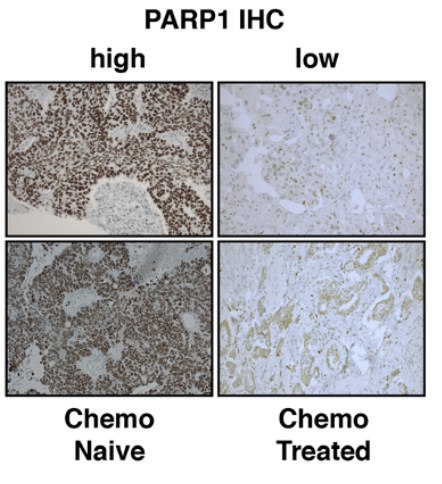

C PARP1 staining

D PARP1 IHC
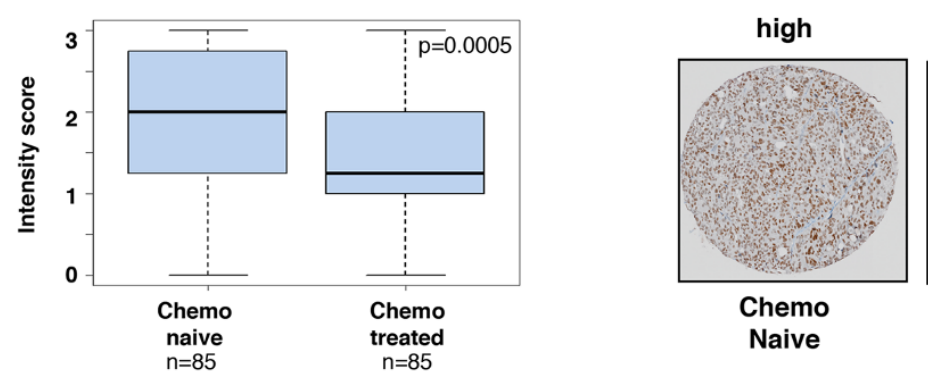

low

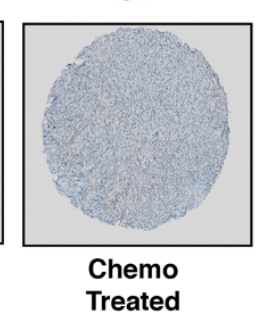

Western Blot

E Quantification of PARP1 in
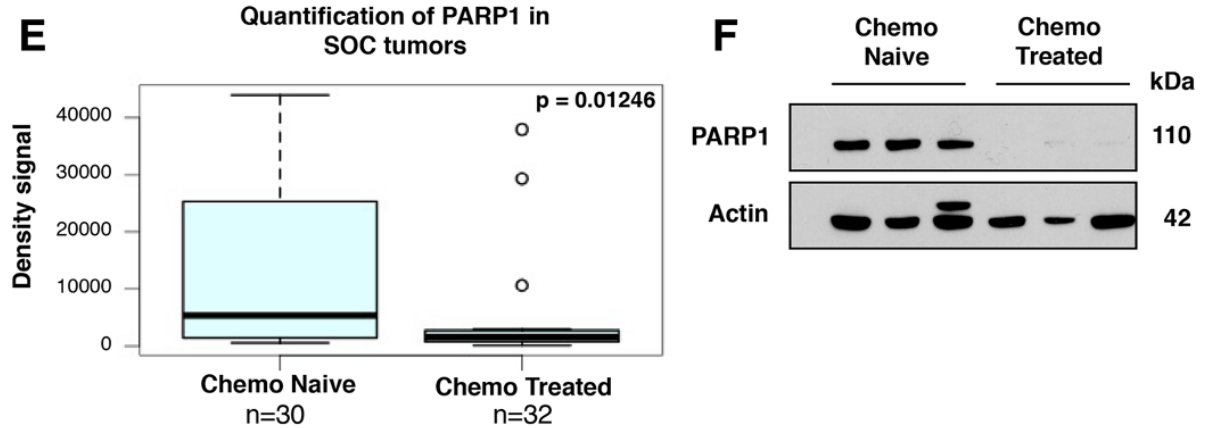

Fig. 2 Chemo-treated tumors have lower levels of PARP1 protein than chemo-naive tumors. a We used 69 HGSC tumors for immunohistochemistry (IHC) staining of PARP1 (training cohort). Each slide score is the product of the percentage of stained tumors cells and the intensity of the staining as described in the "Methods" section. The bar graph represents the percentage of slides with scores in the upper tertile from chemo-naïve and chemo-treated samples. Chi-square test $p<0.001$. b Representative photos of PARP1 IHC from two chemo-naïve tumors and two chemo-treated tumors (20x). c Boxplot showing compilation of PARP1 intensity of staining score for the tissue microarray (TMA). The $p$-value tested whether there was a significant difference in PARP1 staining intensity between the chemo-naïve and chemo-treated tumors and was calculated using a two-tailed Mann-Whitney test. $\mathbf{d}$ Representative TMA cores stain with PARP1 antibody. e PARP1 protein levels in 62 serous ovarian cancer (SOC) tumors (30 chemo-naive and 32 chemo-treated) were quantified with ImageJ and the density signals obtained were used to generate a boxplot. Wilcox Mann-Whitney test gave $p=0.01246$. $\mathbf{f}$ Representative western blots of PARP1 in three chemo-naive tumors and three chemo-treated tumors from cohort shown in $\mathbf{e}$

further validated using western blotting of tumors from 74 unselected patients with ovarian cancer (validation cohort 2). PARP1 protein expression was undetectable in $60 \%$ of the tumors analyzed (Fig. 1d,e), similar to our IHC data and previously published studies $[18,19]$. Of the 100 tumors we analyzed from the 74-patient cohort by western blotting, we further observed $77 \%$ of them had undetectable BRCA1 protein levels (Fig. 1f), and found 
the same ratio of PARP1-expressing and BRCA1expressing tumors as seen in previous studies $[18,19]$. This suggests that samples within this cohort are highly representative of HGSC cohorts examined by other groups. Because of the increasing use of neo-adjuvant therapy, we had the opportunity to evaluate PARP1 expression in cancers before and after chemotherapy and evaluate whether exposure to chemotherapy might play a role in the levels of PARP1 in ovarian tumor. We selected tumor samples from patients with HGSC and compared PARP1 protein levels between chemo-naïve and chemo-treated tumors with standard chemotherapy (carboplatin + taxol) in three patient cohorts: a training cohort (IHC, 69 HGSC samples), validation cohort 1 (IHC, 170 HGSC samples), and validation cohort 2 (western blotting, 74 patients, 62 HGSC samples). We found a significant reduction of PARP1 protein by IHC on the training cohort of 31 chemo-naïve and 38 chemo-treated HGSC (Fig. 2a and Table 1). The proportion of tumors with high PARP1 expression was halved in chemo-treated samples compared to the chemo-naïve tumors (Fig. 2a,b). We next validated these results using validation cohort 1 for a TMA (Fig. 2c,d and Table 2) and validation cohort 2 for western blotting (Fig. 2e,f and Table 3). In both groups, we found a significant reduction of PARP1 expression in the chemo-exposed tumors.

Amongst our samples, we identified 15 matched samples isolated from individual patients from whom a tumor sample was available before and after chemotherapy. Of the patients tested, six showed no PARP1 protein expression. Of the remaining nine, eight had reduced PARP1 protein levels post-chemotherapy (Fig. 3a-c). We further confirmed these results by IHC (Fig. 3d). Although surprising, our results might explain the low, or absent, PARP1 protein seen in a large proportion of the ovarian tumors tested in previous studies [18, 19]. Altogether, these data strongly suggest a suppressive effect of chemotherapy on PARP1 protein expression in solid tumors.

Next, we documented the time between the last chemotherapy treatment and the time at which the biopsy was taken in PARP1-positive and PARP1-negative tumors and found no difference (16.5 days versus 17.0 days). This suggests that the reduction of PARP1 in the tumors was stable and represents a switch in the molecular pathways of the remaining tumor cells post-
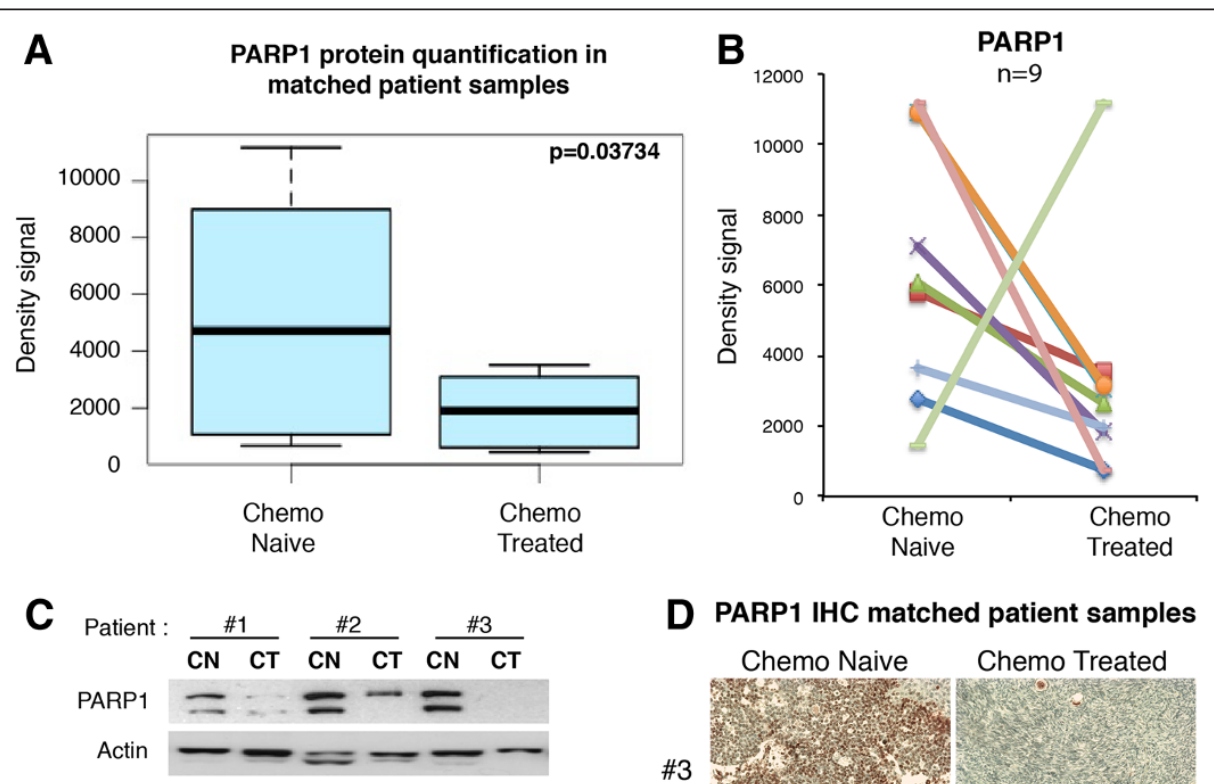

D PARP1 IHC matched patient samples

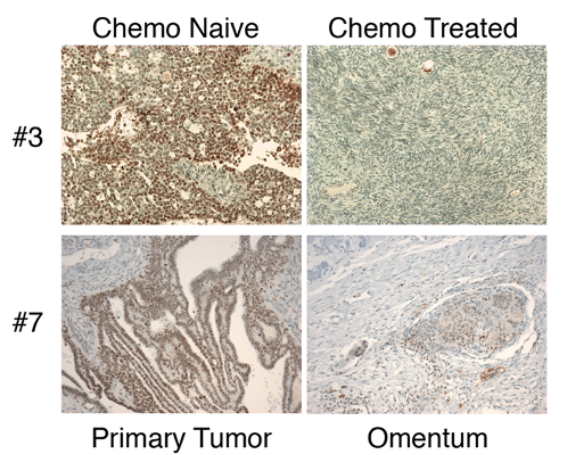

Fig. 3 Chemotherapy decreases PARP1 protein levels in matched patient samples. a PARP1 protein levels in tumor samples from the same 15 patients before and after chemotherapy were quantified with ImageJ and the density signals obtained were used to generate a boxplot. Paired Mann-Whitney test $p=0.03734$. b PARP1 density signals from nine patients with the presence of PARP1 in the tumors before receiving chemotherapy. $\mathbf{c}$ Representative PARP1 and actin western blot of three matched tumor samples before $(C N)$ and after $(C T)$ receiving chemotherapy treatment. $\mathbf{d}$ PARP1

immunohistochemistry $(\mathrm{HC})$ staining in tumor before and after chemotherapy treatment in the same patients (20x) 
chemotherapy or the selection of residual cells that have low PARP1 expression.

In addition, we evaluated whether the expression of PARP1 contributes to platinum sensitivity (defined as no tumor recurrence for greater than 6 months as per "Methods"). These data were available for the 69 tumors within our training cohort. Notably, we found that tumors with high PARP1 expression were significantly more platinum sensitive than low PARP1 expression tumors, independent of previous chemotherapy exposure in the training cohort ( $\chi^{2}$ test, $p<0.001$ ). This may be associated with the fact that PARP1 is an important mediator of cell death in response to stress [20].

We also confirmed using the training cohort that BRCA1-deficient tumors were associated with platinum sensitivity ( $\mathrm{X}^{2}$ test, $p<0.001$ ). Taken together, these findings reinforce the idea that platinum-sensitive BRCA1deficient tumors might respond better to PARP inhibitors when intratumoral PARP1 levels are high.

It is expected that PARP1 expression and a loss of functional BRCA1 are required for sensitivity to PARP inhibitors. In validation cohort 2, the BRCA1-negative, PARP1-positive subset of tumors represented $32.7 \%$ of serous ovarian cancer patients in this study. Strikingly, this number is similar to the proportion of reported positive objective response rate in clinical trials with Olaparib (41 \% [10], $33 \%$ [8], and 25-31 \% [11]). Moreover, no impact of chemotherapy on BRCA1 protein status could be discerned, emphasizing the specificity of the findings. The results suggest that patients receiving PARP inhibitors could be selected not only based on their BRCA1 status, but also for PARP1 protein expression. Such screening is commonplace for other targeted therapies, such as aromatase inhibitors or vemurafenib $[21,22]$, and could be integrated into standard operating procedures for pathologists and oncologists. This concept will be explored in the future within our department. Based on our data, we predict this screening approach would substantially expand the positive objective response rates.

\section{Conclusion}

To our knowledge, this study is the first to probe the effect of chemotherapy treatment on PARP1 protein expression in HGSC. Considering that all patients enrolled in previous clinical trials received several cycles of chemotherapy prior to receiving PARP inhibitor treatment [5, 8-12], these data are likely relevant for guiding future administration of PARP inhibitor therapy. Further studies with independent cohorts are thus warranted to evaluate the effect of PARP1 protein expression on the efficacy of PARP inhibitors. These observations suggest that co-application of PARP inhibitors and chemotherapy as frontline therapy might yield significantly better outcome in HGSC, and could also be explored in other patients with homologous recombination-deficient cancers.

\section{Additional file}

Additional file 1: Figure S1. PARP1 antibody validation. (A) Western blot of PARP1 and actin were performed on OVCAR8 cells infected with shRNA control or directed against PARP1. (B) The same cells used in (A) were paraffin-embedded and IHC staining against PARP1 was performed using the same antibody as in (A). The antibody shows high specificity and sensitivity for PARP1 protein in both techniques. (TIFF $1130 \mathrm{~kb}$ )

\section{Abbreviations}

BRCA1/2: breast cancer 1/2; FIGO: International Federation of Gynecology and Obstetrics; HGSC: high-grade serous carcinoma; IHC: immunohistochemistry; PARP: poly(ADP)-ribose polymerase; TCGA: The Cancer Genome Atlas.

\section{Competing interests}

The authors declare that they have no competing interests.

\section{Authors' contributions}

MM and MCB designed and carried out Western blotting experiments, analyzed survival data, publically available data, and drafted the manuscript. IL gathered the patient's clinical information, produced the tables and contributed to the $\mathrm{IHC}$ analysis. SQ carried out western blot experiments. MP analyzed the $I \mathrm{HC}$ data and confirmed the diagnosis of patients included in the study. DP obtained samples for TMA construction. HF and A-MM-M performed the TMA-related experiments and analysis. MW and WHG designed and supervised the study, and co-edited drafts of the manuscript. All authors read and approved the final manuscript.

\section{Authors' information}

WHG is Professor of Oncology and Obstetrics \& Gynecology at McGill University, Montréal.

\section{Acknowledgements}

This work was funded in part by grants from the Fonds de recherche Québec-Santé, Quebec Breast Cancer Foundation and CIHR to MW. MM is financially supported by Fonds de recherche Québec-Santé and CIHR postdoctoral training grants. The Gyn-Oncology Research Unit is funded by the Montreal-Israel Cancer Research Foundation, the Gloria Shapiro fund, and the Levy Family Fund. Tissue banking was supported by the Réseau de recherche sur le cancer (RRCancer), Fonds de recherche Québec-Santé, affiliated with the Canadian Tumour Repository Network (CTRNet). The various funding agencies were only involved in the material and financial support of the project (including salary and reagents), all the decisions regarding the design, the collection, analysis, and interpretation of the data were made by the authors.

\section{Author details}

${ }^{1}$ Lady Davis Institute for Medical Research, Jewish General Hospital, 3755 Côte Ste, Catherine Road, Montréal, QC H3T 1E2, Canada. ${ }^{2}$ Centre de recherche du Centre hospitalier de I'Université de Montréal/Institut du cancer de Montréal, Montréal, Canada. ${ }^{3}$ Division of Gynecologic Oncology, Jewish General Hospital, McGill University, Montréal, Canada. ${ }^{4}$ Division of Pathology, Jewish General Hospital, Montréal, Canada. ${ }^{5}$ Department of Obstetric-Gynecology, Université de Montréal, Montreal, Canada.

${ }^{6}$ Department of Medicine, Université de Montréal, Montreal, Canada.

Received: 3 June 2015 Accepted: 14 August 2015

Published online: 09 September 2015

\section{References}

1. Jemal A, Siegel R, Ward E, Hao Y, Xu J, Murray T, et al. Cancer statistics, 2008. CA Cancer J Clin. 2008;58:71-96. 
2. Permuth-Wey J, Sellers TA. Epidemiology of ovarian cancer. Methods Mol Biol. 2009;472:413-37.

3. Miki Y, Swensen J, Shattuck-Eidens D, Futreal PA, Harshman K, Tavtigian S, et al. A strong candidate for the breast and ovarian cancer susceptibility gene BRCA1. Science. 1994;266:66-71.

4. Wooster R, Bignell G, Lancaster J, Swift S, Seal S, Mangion J, et al. Identification of the breast cancer susceptibility gene BRCA2. Nature. 1995;378:789-92.

5. Fong PC, Boss DS, Yap TA, Tutt A, Wu P, Mergui-Roelvink M, et al. Inhibition of poly(ADP-ribose) polymerase in tumors from BRCA mutation carriers. N Engl J Med. 2009;361:123-34.

6. Farmer H, McCabe N, Lord CJ, Tutt AN, Johnson DA, Richardson TB, et al. Targeting the DNA repair defect in BRCA mutant cells as a therapeutic strategy. Nature. 2005;434:917-21.

7. Cancer Genome Atlas Research Network. Integrated genomic analyses of ovarian carcinoma. Nature. 2011;474:609-15.

8. Audeh MW, Carmichael J, Penson RT, Friedlander M, Powell B, Bell-McGuinn KM, et al. Oral poly(ADP-ribose) polymerase inhibitor olaparib in patients with BRCA1 or BRCA2 mutations and recurrent ovarian cancer: a proof-of-concept trial. Lancet. 2010;376:245-51.

9. Fong PC, Yap TA, Boss DS, Carden CP, Mergui-Roelvink M, Gourley C, et al. Poly(ADP)-ribose polymerase inhibition: frequent durable responses in BRCA carrier ovarian cancer correlating with platinum-free interval. J Clin Oncol. 2010;28:2512-9.

10. Gelmon KA, Tischkowitz M, Mackay H, Swenerton K, Robidoux A, Tonkin K, et al. Olaparib in patients with recurrent high-grade serous or poorly differentiated ovarian carcinoma or triple-negative breast cancer: a phase 2, multicentre, open-label, non-randomised study. Lancet Oncol. 2011;12:852-61.

11. Kaye SB, Lubinski J, Matulonis U, Ang JE, Gourley C, Karlan BY, et al. Phase II, open-label, randomized, multicenter study comparing the efficacy and safety of olaparib, a poly (ADP-ribose) polymerase inhibitor, and pegylated liposomal doxorubicin in patients with BRCA1 or BRCA2 mutations and recurrent ovarian cancer. J Clin Oncol. 2012;30:372-9.

12. Ledermann J, Harter P, Gourley C, Friedlander M, Vergote I, Rustin G, et al. Olaparib maintenance therapy in platinum-sensitive relapsed ovarian cancer. N Engl J Med. 2012;366:1382-92.

13. Zhao T, Sun Q, del Rincon SV, Lovato A, Marques M, Witcher M. Gallotannin imposes $S$ phase arrest in breast cancer cells and suppresses the growth of triple-negative tumors in vivo. PLoS One. 2014;9, e92853.

14. Balakrishnan SK, Witcher M, Berggren TW, Emerson BM. Functional and molecular characterization of the role of CTCF in human embryonic stem cell biology. PLoS One. 2012;7, e42424.

15. Corder GW, Foreman DI. Nonparametric statistics for non-statisticians: a step-by-step approach. Hoboken, NJ: John Wiley \& Sons, Inc.; 2011.

16. Newell-Litwa K, Salazar G, Smith Y, Faundez V. Roles of BLOC-1 and adaptor protein-3 complexes in cargo sorting to synaptic vesicles. Mol Biol Cell. 2009;20:1441-53.

17. Goncalves A, Finetti P, Sabatier R, Gilabert M, Adelaide J, Borg JP, et al. Poly(ADP-ribose) polymerase-1 mRNA expression in human breast cancer: a meta-analysis. Breast Cancer Res Treat. 2011;127:273-81.

18. Barnett JC, Bean SM, Nakayama JM, Kondoh E, Murphy SK, Berchuck A. High poly(adenosine diphosphate-ribose) polymerase expression and poor survival in advanced-stage serous ovarian cancer. Obstet Gynecol. 2010;115:49-54.

19. Gan A, Green AR, Nolan CC, Martin S, Deen S. Poly(adenosine diphosphateribose) polymerase expression in BRCA-proficient ovarian high-grade serous carcinoma; association with patient survival. Hum Pathol. 2013;44:1638-47.

20. Zong WX, Ditsworth D, Bauer DE, Wang ZQ, Thompson CB. Alkylating DNA damage stimulates a regulated form of necrotic cell death. Genes Dev. 2004; 18:1272-82

21. Baum M, Budzar AU, Cuzick J, Forbes J, Houghton JH, Klijn JG, et al. Anastrozole alone or in combination with tamoxifen versus tamoxifen alone for adjuvant treatment of postmenopausal women with early breast cancer: first results of the ATAC randomised trial. Lancet. 2002;359:2131-9.

22. Bollag G, Hirth P, Tsai J, Zhang J, Ibrahim PN, Cho H, et al. Clinical efficacy of a RAF inhibitor needs broad target blockade in BRAF-mutant melanoma. Nature. 2010;467:596-9.

\section{Submit your next manuscript to BioMed Central and take full advantage of:}

- Convenient online submission

- Thorough peer review

- No space constraints or color figure charges

- Immediate publication on acceptance

- Inclusion in PubMed, CAS, Scopus and Google Scholar

- Research which is freely available for redistribution

Submit your manuscript at www.biomedcentral.com/submit 